\title{
A systematic review of the long-term efficacy of low-intensity shockwave therapy for vasculogenic erectile dysfunction
}

\author{
Oliver Brunckhorst ${ }^{1} \cdot$ Lauren Wells $^{1} \cdot$ Fiona Teeling $^{1} \cdot$ Gordon Muir $^{1} \cdot$ Asif Muneer $^{2} \cdot$ Kamran Ahmed $^{1,2,3}$
}

Received: 19 January 2019 / Accepted: 7 March 2019 / Published online: 22 March 2019

(c) The Author(s) 2019

\begin{abstract}
Purpose To look at the evidence base for LISWT as a treatment modality for vasculogenic erectile dysfunction, focusing on the long-term outcomes at over 6 months following treatment.

Methods A systematic literature search was conducted utilising MEDLINE and Scopus databases from 2010 to September 2018 by two independent reviewers. Outcome measures extracted for long-term efficacy included International Index of Erectile Function scores and Erection Hardness Scores. Subgroup analysis for LISWT effectiveness included age, PDE5i responsiveness, presence of vascular co-morbidities and smoking status.

Results The search identified eleven studies, representing a total of 799 patients. Nine studies found a significant improvement in erectile function after LISWT at 6-month follow-up (median IIEF-EF improvement in 5.3 at 6 months). However, of five studies assessing erectile function at 12 months; two identified a plateauing of results, with three a deterioration (IIEFEF score changes of -2 to 0.1 from 6 months). Erectile function did, however, remain above baseline results in all of these studies. Subgroup analysis revealed increasing age to reduce the response to LISWT treatment. Whilst ED severity, PDE5i responsiveness and co-morbidities potentially influence effectiveness, results are still inconsistent.

Conclusions LISWT may be a safe and acceptable potential ED treatment with demonstrated benefits at 6 months. There is some question regarding efficacy deterioration beyond this, but there is still a demonstrated benefit seen even at 12 months post treatment. However, quality of evidence remains low with larger multiinstitutional studies required, standardising confounders such as shockwave administration and oral medication use.
\end{abstract}

Keywords Erectile dysfunction · Vasculogenic impotence · Extracorporeal shockwave therapy

Electronic supplementary material The online version of this article (https://doi.org/10.1007/s11255-019-02127-z) contains supplementary material, which is available to authorized users.

Kamran Ahmed

kamran.ahmed@kcl.ac.uk

Oliver Brunckhorst

oliver.brunckhorst@kcl.ac.uk

Lauren Wells

lauren.wells@kcl.ac.uk

Fiona Teeling

fiona.teeling@kcl.ac.uk

Gordon Muir

gordon.muir@kcl.ac.uk

\section{Introduction}

Atherosclerosis of penile arteries and endothelial dysfunction, known as vasculogenic erectile dysfunction (ED), is the cause of ED in $40 \%$ of men over the age of fifty [1]. There is currently no known long-lasting or curative treatment for vasculogenic ED [2]. At present both AUA and EAU guidelines for

Asif Muneer

asif.muneer@nhs.net

1 MRC Centre for Transplantation, Guy's Hospital Campus, King's College London, King's Health Partners, London SE1 9RT, UK

2 Department of Urology, University College Hospital, University College London Hospitals NHS Foundation Trust, London, UK

3 Department of Urology, King's College Hospital, London, UK 
the treatment of vasculogenic ED recommend initial lifestyle changes to address modifiable risk factors, followed by oral phosphodiesterase 5 inhibitors (PDE5is) as first line medical management. However, only $80 \%$ of patients respond to PDE5is as its mechanism of action requires both intact nerves and a basic level of endothelial function [3]. PDE5is are contraindicated in patients using nitrate therapy, so a significant proportion of patients with Vascular ED are forced into second and third line treatment options [4]. Alternative treatment options to PDE5is include vacuum erection devices which are simple to use but have variable patient satisfaction rates [5], intracavernosal injections and topical prostaglandin E1 analogues (Alprostadil). Patients unresponsive to first and second line treatments may progress to surgical management with penile prosthesis [4].

The precise mechanism of action of low-intensity shockwave therapy (LISWT) is not fully understood; however, it is believed that the compression and subsequent negative pressures created from the shockwave energy, the so-called cavitation phenomenon, is an important factor [6]. These tensile forces lead to shear stress on cell membranes which have been shown to have the potential to treat the underlying cause of vascular ED by prompting increased expression of vascular endothelial growth factor (VEGF) [7], recruitment of perivascular stem cells and recruitment of endothelial progenitor cells [8], resulting in neovascularisation of penile arteries. Furthermore, shockwaves may also improve nerve regeneration as seen in animal studies, due to a hypothesised increasing in the ability of injured axons to repair and Schwann cell proliferation [9] which may be useful in ED caused by neurovascular aetiologies. LISWT is unlike any of the currently offered treatment options as it could provide men with a natural erection by treating the underlying pathophysiology, rather than treating the symptoms.

However, the evidence for its use is still debated at present, lacking Food and Drug Administration (FDA) approval for $\mathrm{ED}$, and is still considered experimental by organisations such as the Sexual Medicine Society of North America. Additionally, there has so far largely been a focus on the short-term efficacy of LISWT as a treatment mobility. This review of the literature therefore aimed to:

1. Assess the current evidence base focusing on the longterm outcomes at over 6 months of using LISWT as a treatment modality for vascular ED.

2. Identify if any cohorts of patients with demonstrated improved long-term efficacy of treatment after LISWT

\section{Materials and methods}

This review was performed following guidelines defined in the Preferred Reporting Items for Systematic Reviews and Meta-Analyses (PRISMA) statement [10]. The review was prospectively registered, PROSPERO registration number: CRD42018112789.

\section{Study eligibility criteria}

Original research articles utilising LISWT to treat vasculogenic ED, with a minimum follow-up period of 6 months for their cohorts, were included. Study types included were randomised controlled trials (RCTs) as well as prospective and retrospective single arm experimental studies. Exclusion criteria were articles not utilising either International Index of Erectile Function-Erectile Function Domain (IIEF-EF) or Erection Hardness Score (EHS) as an outcome parameter and studies published before 2010 (the first trial for LISWT in vasculogenic ED was conducted in 2010 [2]). Furthermore, animal studies, case studies, reviews, studies using LISWT for non-vasculogenic ED and studies unavailable in the English language were all excluded from the review.

\section{Information sources and search}

Electronic databases, MEDLINE (via PubMed) and Scopus, were systematically searched for research articles from January 2010 to September 2018. A combination of MeSH terms and key terms was used ('Low-Intensity Shockwave Therapy' OR 'Pulsed Ultrasound' OR 'Low Intensity Ultrasound' OR 'Shockwave' OR 'Shock wave') AND ('Erectile Dysfunction' OR 'ED' OR 'Sexual Dysfunction'). In addition, a thorough reference review of identified articles was conducted, to ensure that all relevant articles were included. The grey literature was searched via abstracts on Scopus and ongoing clinical trials in Cochrane Library and ClinicalTrials.gov, with authors contacted for any available preliminary data.

\section{Study selection}

The search was conducted independently by two reviewers (OB and LW) to identify potentially relevant articles. Title and abstract screening was conducted, with full-text articles subsequently screened along inclusion criteria for inclusion into qualitative analysis. Discrepancies between reviewers were discussed until $100 \%$ agreement was achieved.

\section{Data collection and data items}

Data extraction was independently conducted by two reviewers (OB and $\mathrm{LW})$. Specific data were extracted from 
all studies such as study type, number of participants, participant demographics and LISWT treatment regimen. The primary outcome measure extracted for clinical efficacy included erectile function measures such as IIEF-EF or EHS scores after LISWT at long-term follow-up of over 6 months. This included both raw questionnaire score improvements, percentage improvements and also study defined success rates as per score improvements. Additionally, subgroup analysis of LISWT effectiveness was conducted via assessment of population cohorts including age, PDE5i responsiveness, presence of vascular co-morbidities and smoking status.

\section{Risk of bias in individual studies and across studies}

The internal validity of each individual article was assessed using the Cochrane Risk of bias tool and with further qualitative analysis for randomised controlled trials and non-randomised studies, respectively [11]. Non-randomised studies were assessed qualitatively by authors critically appraising the methodology, as per definitions in Online Resource 1. Bias across studies was assessed via the GRADE tool in order to provide a recommendation from our review for each individual outcome measure [12].

\section{Results}

\section{Study selection}

A total of 521 articles were identified through the literature search. Duplicate removal and initial screening excluded 434 articles. Of the 87 full-text articles assessed for eligibility, a final eleven articles were included in the review (Fig. 1).

\section{Study characteristics and result synthesis}

Of the eleven articles, five were RCTs and six were nonrandomised (Table 1). Three of the RCTs were placebo-controlled; the others compared two different treatment groups. The total number of patients investigated across all studies was 799 patients.

\section{Long-term efficacy of LISWT}

Nine studies of the eleven studies [2,13-20] found a statistically significant increase in erectile function utilising either IIEF or EHS scores after LISWT at over 6-month follow-up (median IIEF-EF score improvement from baseline at 6 months 5.3, range 2.6-10.7). None of these studies demonstrated a decrease in erectile function below baseline post intervention (Fig. 2). However, the results from two randomised, sham-controlled studies, Fojecki et al. and Olsen et al., did not reach the authors' set threshold for significance at follow-ups of over 6 months [21, 22]. The effects of LISWT were followed up for 24 months in one study, 12 months in four studies and 6 months in the remaining six studies. When assessing studies with follow-ups of greater than 6 months, there appeared to be limited improvement in IIEF scores beyond this time period (change in IIEF-EF scores of between -2 and 0.1 ). No studies identified an ongoing improvement at 12 months when compared to 6 months with two studies demonstrating plateauing of IIEF scores $[15,18]$. Three studies showed there was a gradual diminishing effect of effectiveness of LISWT beyond 6 months; however, scores remained at above baseline erectile function in all cases $[13,16,21]$. The largest of these studies demonstrating a gradual decline was conducted by Kitrey et al. This prospective single-armed trial of 156 patients demonstrated an initial response rate of $63.5 \%$ at 1 month, decreasing to $42.9 \%$ at 12 months and declining to just $34 \%$ at 2-year follow-up.

\section{Effect of LISWT on specific population cohorts}

Subgroup analysis demonstrated conflicting findings between studies. Whilst the majority of studies have not been powered to draw conclusions based on specific subgroups, trends in results were seen. Age, PDE5i responsiveness, presence of vascular co-morbidities and smoking status have all been proposed to impact the efficacy of LISWT treatment.

Two out of three studies assessing age specifically identified a reduced effectiveness of LISWT with increasing age $[14,17]$. These studies identified that younger age was a statistically significant predictor for improved treatment responsiveness and increasing age ( $>65$ years) and vascular co-morbidities shortened the duration of LISWT effects in comparison to younger, healthier patients. However, in contrast the study conducted by Bechara et al. saw no statistically significant difference in age, duration of ED or co-morbidities when comparing LISWT responders to nonresponders [18].

ED severity appears to have contrasting effects on efficacy. Whilst the study conducted by Bechara et al. identified that patients with severe ED responded better to LISWT with the greatest IIEF-EF point increase, Kitrey and colleagues found that the duration of treatment effect was reduced in patients with severe ED at 24-month follow-up [13, 18]. Additionally, when considering duration of ED symptoms, Reisman et al. identified a negative effect on LISWT responsiveness with increasing duration of symptoms, finding the average ED duration to be 3.5 years longer in LISWT non-responders than responders [20]. PDE5i response was seen to be important in a 


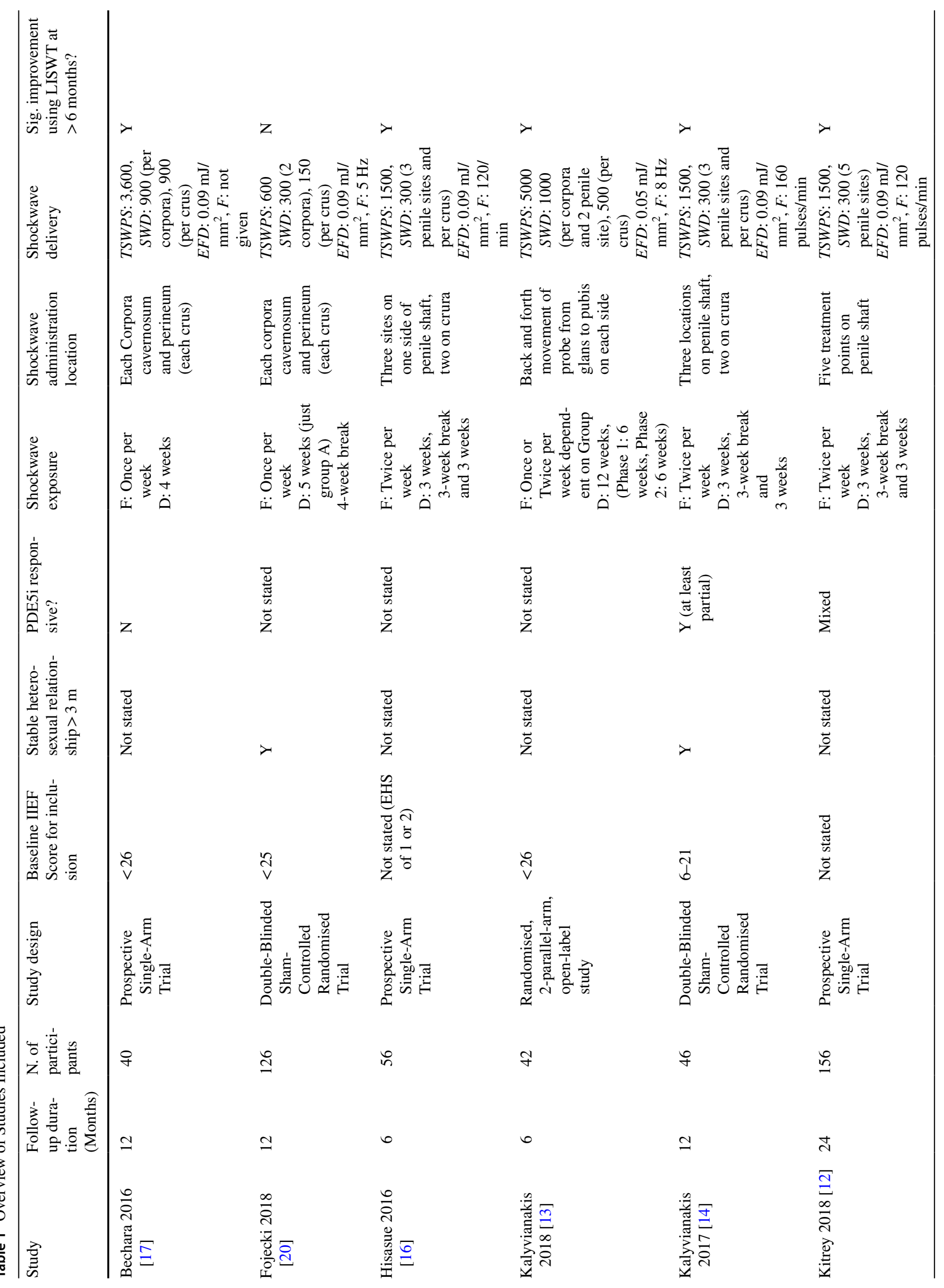




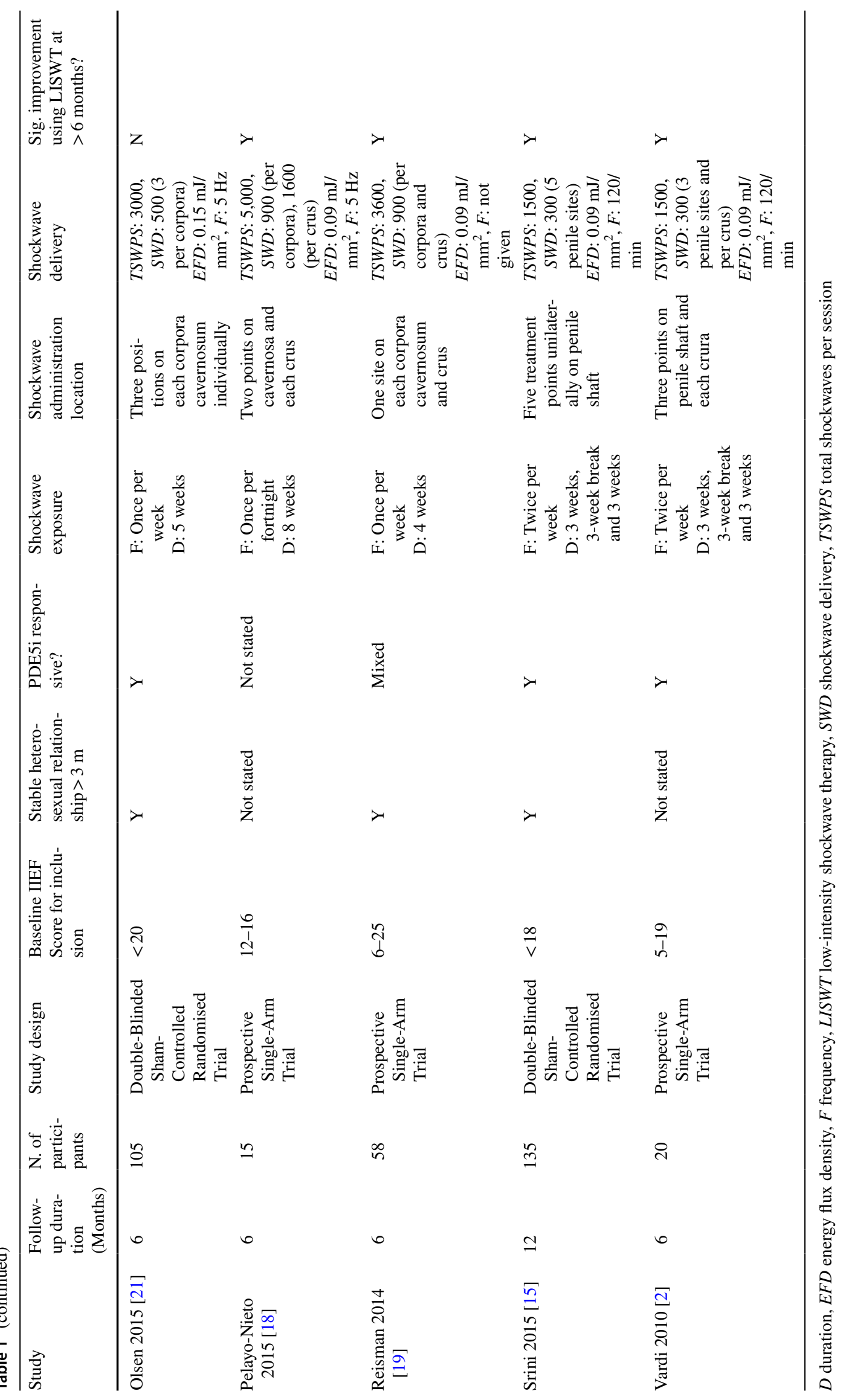




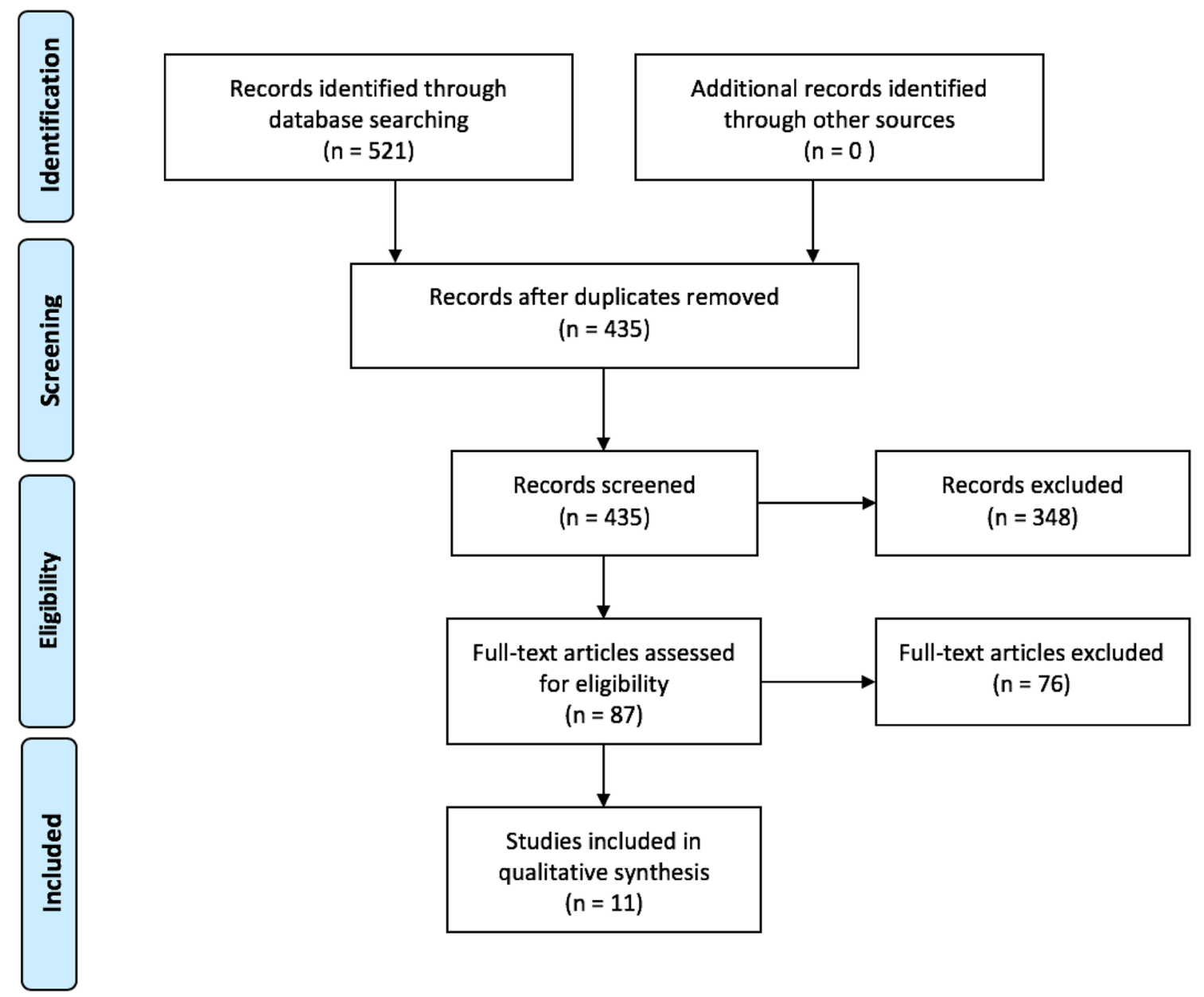

Fig. 1 PRISMA flow chart for article selection

single study where PDE5i responders were statistically more likely to receive benefit from LISWT, with longer duration of efficacy in this cohort [14].

Two studies assessed the effect of vascular risk factors on LISWT effectiveness. In a comparison of patients with at least one vascular co-morbidity (cardiovascular disease, hypertension, high cholesterol), to those with none, Reisman et al. identified lower success rates in those with co-morbidities ( $76.2 \%$ vs $93.7 \%$, respectively) [20]. Smoking status was seen to negatively impact the success of LISWT in one study, with patients possessing a smoking index of less than 20 having a statistically significant chance of improving erectile function $(91 \%$ vs. 50\%) [19]. Finally, the presence of diabetes demonstrated mixed results. Whilst one study demonstrated success rates which were $25 \%$ lower in diabetic patients, with shorter duration of treatment effects [20], Pelayo-Nieto et al. contrastingly identified that diabetic patients demonstrated an improved clinical response to LISWT (62\% vs $47 \%$ ) [19].

\section{Quality assessment of articles}

There are still limited number of studies assessing longterm follow-up after LISWT with predominantly nonrandomised trials present. Risk of bias assessment of randomised trials (Online Resource 2) showed the largest concern to be regarding selection bias, introduced by attrition of study participants. Whilst higher attrition rates are expected due to the longer follow-up in our selected articles, unusually high dropout rates in some studies such as Srini and Fojecki et al. (over 20\%) which was identified to be more heavily weighted towards the placebo or the 5 -week treatment group [16, 21]. This could skew results towards those receiving the treatment, generating falsepositive results. Additionally, the five randomised trials demonstrated small numbers of total participants, with all being single centre trials. There are finally concerns regarding the sham or double blinded nature of the trials. It is difficult to ensure true blinding in these circumstances with many of the trials identifying no benefit at all 


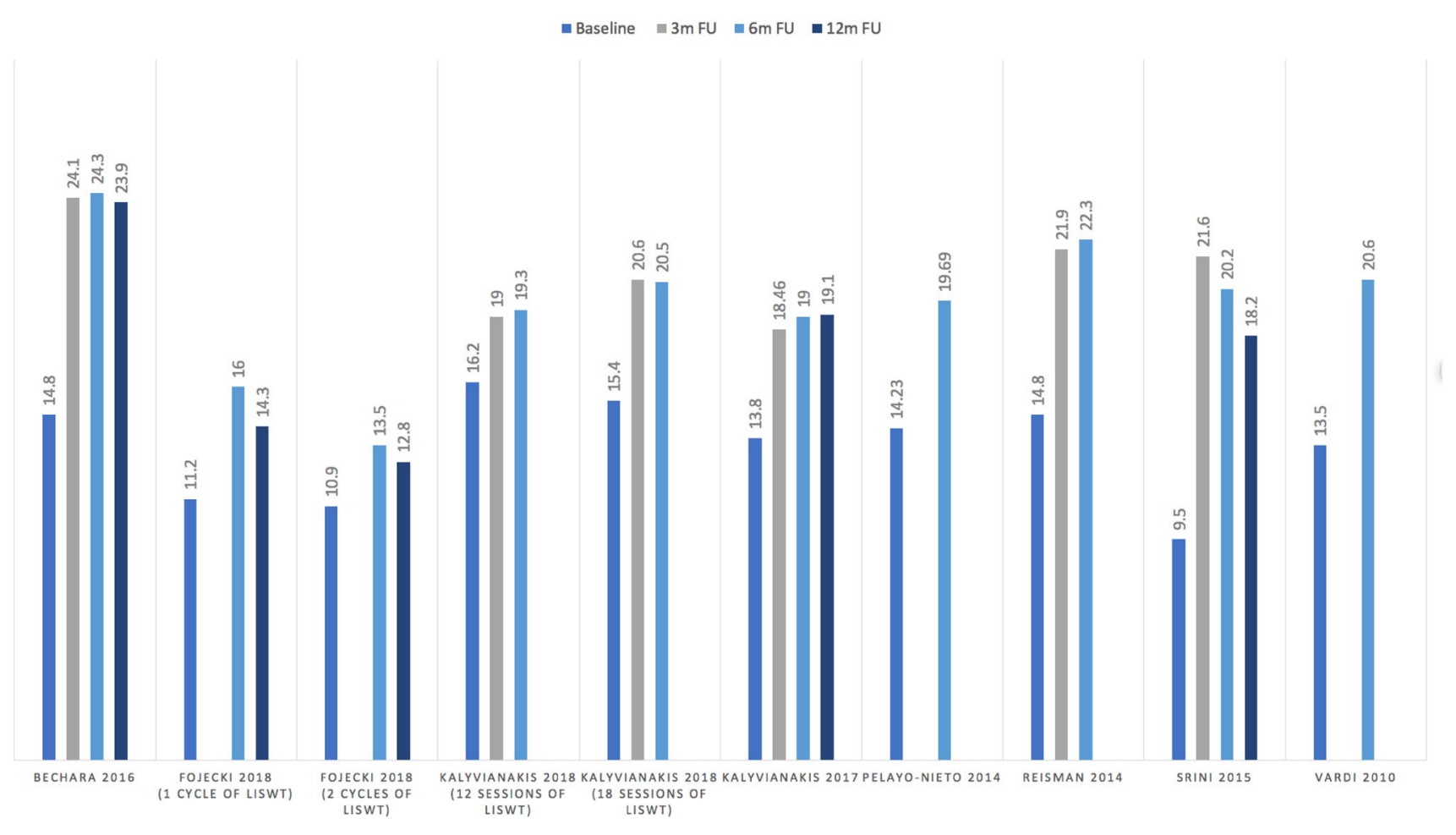

Fig. 2 Long-term efficacy of LISWT based on IIEF-EF scores at baseline, 3, 6 and 12 months

from sham treatment which is unexpected as some placebo effect is expected.

PDE5i use prior and during treatment is currently variable. Whilst the majority of studies included a 4-week 'washout period' without PDE5i use, this was not consistent across all studies, with additional variation surrounding ongoing PDE5i use and timing of restarting this. Four studies allowing participants to resume PDE5i use from 1-month post-LISWT [2, 17, 20, 21], and one study keeping patients on treatment throughout entire treatment duration [18]. This is important as this produces as a confounder which may explain differences in long-term efficacy post-LISWT. Finally, an extremely large variation in administration protocols was identified. Individual studies varied significantly in terms of shockwaves delivered per session, time between sessions and even sites of administration as demonstrated in Table 1 . This presents a limitation towards external validity of the studies in view of effect sizes for long-term erectile function post-LISWT.

\section{Comment}

This systematic review assesses the long-term effect of LISWT at over 6 months on vasculogenic ED patients. Out of the eleven papers identified, nine demonstrated a statistically significant improvement in erectile function at 6-month follow-up. However, these studies show that beyond 6 months there is no ongoing improvement in erectile function seen. Three out of five studies demonstrated a gradual decline in erectile function with two showing a plateauing of results. However, it is important to note that IIEF-EF scores in all studies remained significantly above baseline functional scores, demonstrating benefit even at 12 -month posttreatment. This is likely secondary to the ongoing underlying vascular progression of disease, with LISWT having no impact on comorbidities or progressive atherosclerotic disease affecting the cavernosal tissue [13]. Assessment of the overall quality of the evidence for long erectile function improvement via the GRADE protocol demonstrates that current recommendation for use remains low at present (Online Resource 3). This is due to the predominantly nonrandomised evidence base currently present with trials presenting small patient numbers, single institutions and methodological concerns regarding the double-blind sham trials.

Subgroup analysis assessing individual cohorts of patients for LISWT effectiveness yielded varying results. Two studies suggest that younger patients may be more likely to benefit from prolonged benefits in erectile function. This is hypothesised to be secondary to less structural cavernosal and ultra-structural damage present, with greater VEGF receptor activity resulting in a greater biological response from treatment $[14,17]$. This has led to suggestions that LISWT may have a role in early intervention, or 
even prophylactic treatment in high-risk patients, thereby preventing irreversible vascular changes [14, 23]. However, at present the objective evidence for this is non-existent. Similarly, it would be expected that patients with severe ED would see a reduced effectiveness of treatment secondary to increased ultra-structural damage. Whilst previous findings in short-term follow-up have supported this [24], our review has identified conflicting evidence in the long term, with no clear relationship of ED severity or duration to clinical efficacy.

The evidence assessing cardiovascular co-morbidities and risk factors on treatment effect in the long term is limited. There is contrasting evidence assessing diabetic patients with further review certainly needed. Furthermore, whilst smoking and presence of other cardiovascular risk factors appear to negatively impact the efficacy of treatment, these results are restricted to a single study only in the long term [20]. Similarly, whilst PDE5i responders and naïve patients have previously been identified as positive predictive markers for treatment success in short term studies [24], this cannot be determined for long-term studies yet. Only a single study demonstrated improved efficacy; however, this was not statistically significant. Therefore, at present there is no concrete evidence for any subgroup identified as a predictive marker for long-term successful treatment.

This is the first review, to our knowledge, to specifically assess the long-term efficacy of LISWT for vasculogenic ED. It provides important evidence to demonstrate that there appears to be a lasting effect of erectile function improvement at 6 months for patients which either plateau or may gradually deteriorate towards 12 months post-treatment. This is clinically important, providing urologists evidence for treatment, but additionally offers evidence for frank discussion regarding expectations of erectile function beyond 6-month post-treatment. However, as previously mentioned due to varying study methodologies this review highlights to researchers further areas of research to increase the evidence base surrounding LISWT use.

Whilst there is clinically relevant data currently available, there are several concerns regarding current methodology of identified trials which require standardisation in future studies. It is clear larger studies which are multi-institutional and multi-national are required in the first instance to increase external validity of results. Further to this, PDE5is use prior and post-treatment is extremely variable in the literature. Whilst a washout period and limited PDE5i use may improve the results obtained, it could be argued that future research should focus on more real-life applicability by maintenance of medical therapy concurrently to LISWT. Additionally, future research must standardise administration of LISWT, in particular with regard to the device utilised, treatment delivery in terms of sessions and duration as well as location of administration with current positioning widely varied in the studies identified. In terms of outcome measures utilised, there is a need for greater objective parameters through penile haemodynamic studies concurrently to subjective measures such as IIEF-EF and EHS scores, which can be heavily influenced by other factors such as sexual partners, lifestyle, life events, psychology and comorbidities [13]. Finally, this review has identified a need for longer followup at 12 months and beyond to assess the ongoing longevity of treatment efficacy.

As is the case with any systematic review, this review has its limitations. These are largely arising from the data set available with limitations in the number of trials which are available with few randomised studies identified assessing long-term outcomes specifically. Additionally, several methodological concerns and variations as previously mentioned are present. This is particularly true when considering treatment administration and concurrent therapy, meaning that generalisability of results must be considered, and hence, standardised recommendations could not be made. When this variability in methodology was combined with the lack of randomised trials reporting standardised outcome measures such as IIEF-EF and the large heterogeneity of results seen when statistical pooling of randomised studies was attempted, meant that any meaningful statistical assessment of data via a meta-analysis was not feasible. Finally, there is always the possibility of missed studies which could affect current recommendation; however, the risk of this was minimised via a comprehensive search strategy and searching both grey and current literature.

\section{Conclusions}

This systematic review identifies that LISWT offers a treatment modality which improves erectile function, with results lasting to over 6 months. There appears to be some limitation of ongoing benefit beyond this at 12 months with either plateauing or even reduction in functional outcomes at this time. Increasing age appears to reduce responsiveness to LISWT treatment in long-term follow-up studies. Furthermore, ED severity, PDE5i responsiveness and co-morbidities may also influence its effectiveness; however, results are inconsistent at present. Whilst LISWT may be a safe and acceptable long-term ED treatment modality, it is clear further investigation is still needed through larger and more standardised trials to improve its evidence base.

Acknowledgements Oliver Brunckhorst acknowledges research support from the National Institute for Health Research and the MRC Centre for Transplantation at King's College London. Asif Muneer is supported by the National Institute for Health Research Biomedical Research Centre University College London Hospital. Kamran Ahmed acknowledge receiving research support from The Urology Foundation, 
the Royal College of Surgeons of England, the Pelican Group and the MRC Centre for Transplantation at King's College London.

Funding This study did not receive specific funding by any bodies.

\section{Compliance with ethical standards}

Conflict of interest All other authors have no conflicts of interest to make.

Ethical approval This article does not contain any studies with human participants performed by any of the authors.

Open Access This article is distributed under the terms of the Creative Commons Attribution 4.0 International License (http://creativeco mmons.org/licenses/by/4.0/), which permits unrestricted use, distribution, and reproduction in any medium, provided you give appropriate credit to the original author(s) and the source, provide a link to the Creative Commons license, and indicate if changes were made.

\section{References}

1. Wyllie MG (2005) The underlying pathophysiology and causes of erectile dysfunction. Clin Cornerstone 7:19-27

2. Vardi Y, Appel B, Jacob G et al (2010) Can low-intensity extracorporeal shockwave therapy improve erectile function? A 6-month follow-up pilot study in patients with organic erectile dysfunction. Eur Urol 58:243-248

3. Hatzimouratidis K, Salonia A, Adaikan G et al (2016) Pharmacotherapy for erectile dysfunction: Recommendations from the Fourth International Consultation for Sexual Medicine (ICSM 2015). J Sex Med 13:465-488

4. NHS England (2016) Clinical Commissioning Policy: Penile Prosthesis surgery for end stage erectile dysfunction. NHS England. https://www.england.nhs.uk/wp-content/uploads/2018/07/Penil e-prostheses-for-end-stage-erectile-dysfunction.pdf. Accessed 28 November 2018

5. Hatzimouratidis K, Giuliano F, Moncada I et al (2018) EAU guidelines: male sexual dysfunction. Arnhem: EAU Guidelines Office

6. Wang HJ, Cheng JH, Chuang YC (2017) Potential applications of low-energy shock waves in functional urology. Int J Urol 24:573-581

7. Nishida T, Shimokawa H, Oi K et al (2004) Extracorporeal cardiac shock wave therapy markedly ameliorates ischemia-induced myocardial dysfunction in pigs in vivo. Circulation 110:3055-3061

8. Aicher A, Heeschen C, Sasaki K et al (2006) Low-energy shock wave for enhancing recruitment of endothelial progenitor cells: a new modality to increase efficacy of cell therapy in chronic hind limb ischemia. Circulation 114:2823-2830

9. Hausner T, Pajer K, Halat G et al (2012) Improved rate of peripheral nerve regeneration induced by extracorporeal shock wave treatment in the rat. Exp Neurol 236:363-370
10. Moher D, Liberati A, Tetzlaff J et al (2009) Preferred reporting items for systematic reviews and meta-analyses: the PRISMA statement. Ann Intern Med 151:264-269

11. Higgins JP, Altman DG, Gøtzsche PC et al (2011) The Cochrane Collaboration's tool for assessing risk of bias in randomised trials. BMJ 343:d5928

12. Guyatt G, Oxman AD, Akl EA et al (2011) GRADE guidelines: 1 . Introduction-GRADE evidence profiles and summary of findings tables. J Clin Epidemiol 64:383-394

13. Kitrey ND, Vardi Y, Appel B et al (2018) Low intensity shock wave treatment for erectile dysfunction-how long does the effect last? J Urol 200:167-170

14. Kalyvianakis D, Memmos E, Mykoniatis I et al (2018) Low-intensity shockwave therapy for erectile dysfunction: a randomized clinical trial comparing 2 treatment protocols and the impact of repeating treatment. J Sex Med 15:334-345

15. Kalyvianakis D, Hatzichristou D (2017) Low-intensity shockwave therapy improves hemodynamic parameters in patients with vasculogenic erectile dysfunction: a triplex ultrasonography-based sham-controlled trial. J Sex Med 14:891-897

16. Srini VS, Reddy RK, Shultz T et al (2015) Low intensity extracorporeal shockwave therapy for erectile dysfunction: a study in an Indian population. Can J Urol 22:7614-7622

17. Hisasue $S$, China $T$, Horiuchi $A$ et al (2016) Impact of aging and comorbidity on the efficacy of low-intensity shock wave therapy for erectile dysfunction. Int J Urol 23:80-84

18. Bechara A, Casabé A, De Bonis W et al (2016) Twelve-month efficacy and safety of low-intensity shockwave therapy for erectile dysfunction in patients who do not respond to phosphodiesterase type 5 inhibitors. Sex Med 4:e225-e232

19. Pelayo-Nieto M, Linden-Castro E, Alias-Melgar A et al (2015) Linear shock wave therapy in the treatment of erectile dysfunction. Actas Urol Esp 39:456-459

20. Reisman Y, Hind A, Varaneckas A et al (2014) Initial experience with linear focused shockwave treatment for erectile dysfunction: a 6-month follow-up pilot study. Int J Impot Res 27:108-112

21. Fojecki GL, Tiessen S, Osther PJS (2018) Effect of linear lowintensity extracorporeal shockwave therapy for erectile dysfunction-12-month follow-up of a randomized, double-blinded, shamcontrolled study. Sex Med 6:1-7

22. Olsen AB, Persiani M, Boie S et al (2015) Can low-intensity extracorporeal shockwave therapy improve erectile dysfunction? A prospective, randomized, double-blind, placebo-controlled study. Scand J Urol 49:329-333

23. Musicki B, Bella AJ, Bivalacqua TJ et al (2015) Basic science evidence for the link between erectile dysfunction and cardiometabolic dysfunction. J Sex Med 12:2233-2255

24. Fode M, Hatzichristodoulou G, Serefoglu EC et al (2017) Lowintensity shockwave therapy for erectile dysfunction: is the evidence strong enough? Nat Rev Urol 14:593-606

Publisher's Note Springer Nature remains neutral with regard to jurisdictional claims in published maps and institutional affiliations. 